\title{
Interactive comment on "Multi-model study of mercury dispersion in the atmosphere: Vertical distribution of mercury species" by Johannes Bieser et al.
}

\section{Anonymous Referee \#1}

Received and published: 29 December 2016

The manuscript examines the vertical profiles and inter-hemispheric gradients of mercury species observed during three recent aircraft campaigns using an ensemble of seven atmospheric mercury models. The study also evaluates the response of the models to the simulated chemistry and emission speciation, two processes that are poorly understood. This study beings together a large set of models and observations to understand these processes and is an important contribution to the field. My comments on the manuscript are as follows:

General comments: 1) A large portion of the manuscript is focused on comparing model results to the observations, but the authors do not provide any numerical measure of agreement between the model and observations. There are several qualitative 
comparisons, but the lack of numerical comparison makes it very difficult for the readers to draw their own conclusions. I recommend the authors include one or more of the standard metrics (mean bias, mean error, correlation coefficient, etc.) for modelobservation comparisons.

2) The manuscript lacks a discussion of the representativeness of the observations. I understand that these are the best observations we have, but, as the authors also point out on Line 116-117, aircraft based observations are not representative. Yet they seem to ignore the limited temporal and spatial coverage of the observations when they construct vertical profiles of TM and GEM for the northern mid-latitudes (Fig. 1). It is not clear how these profiles were calculated. Secondly, it is not clear for what time period was simulated by the models, how were the models sampled, and what steps were taken to address the issue of representativeness when making comparisons between the model and the observations.

3) It seems to me that the manuscript was not thoroughly proof-read before publication in ACPD. There are several minor errors that often are distracting. For example, the citation 'Lyman and Jaffe, 2012', was cited at times as 'Lyman and Jaffe, 2011', 'Lyman and Jaffe et al., 2012', 'Lymann and Jaffe, 2012', and 'Jaffe and Lyman, 2012'). There are also a few instances where abbreviations were used without prior definitions. For example, in the main text RM was used first on Line 515, but not defined until Line 663 , while in the abstract it was referred to as oxidized mercury, but in the main text as reactive mercury. I recommend that the manuscript be thoroughly proof-read before final submission.

Specific comments:

1) Sect. 2.3: What was the spin-up period for the sensitivity simulations?

2) Lines 452-457: The underestimte in GOM concentrations seems to be related to the ambient absolute humidity and ozone concentrations and is likely not systematic, as stated by the authors. 
3) Line 543: Do the authors mean measured 'variability' instead of 'uncertainty'? Same for Lines 699, 797.

4) Line 567: It is stated that CCLM-CMAQ has the tropopause as its upper boundary, but Fig. 2 shows model values for CCLM-CMAQ upto $18 \mathrm{~km}$.

5) Line 580: 'Linear TM'. This term is not clear.

Interactive

comment

6) Lines 910-920: The authors interpret the high RM above $6 \mathrm{~km}$ as being related to stratospheric transport of $\mathrm{Br}$ and cite Gratz et al. (2015). However, Gratz et al. (2015) did not find evidence of stratospheric intrusion, and the authors' conclusions seem contradictory to that study. Is it possible to reconcile these two interpretations?

7) Line 927: 'OH seems a plausible explanation'. How about $\mathrm{O} 3$ ? The models with $\mathrm{O} 3$ chemistry had better agreement with the observed concentrations for the NOMADSS profile.

8) Lines 940-942: I am not sure how the higher effective height of emissions would affect GEM concentrations in the upper troposphere.

9) Table 1: Is $\mathrm{OH}$ an aqueous phase oxidant of GEM in the GEOS-Chem model?

10) Table 2: Since the results of the sensitivity simulations were not available for all models, I suggest adding a column specifying which models participated in each sensitivity run.

11) Title: Perhaps the title should contain "Vertical and inter-hemispheric distribution of mercury species".

Technical comments:

1) Lines 83-84: Grammar

Printer-friendly version

2) Lines 98-102: Grammar

Discussion paper

3) Line 143: Spelling, 'Woll' 
4) Line 144: What does DOHGS stand for?

5) Line 620: '14th June' seems to be typo.

6) Line 665: Do the authors mean equilibrium between GOM and PBM?

7) Table 1: No emission speciation information for WRF-Chem

8) Table 1: No reference for natural emissions for the first six models.

9) Table 1: Missing footnote 'a'.

10) Table 2: Refers to the emission set as UNEP2010, while Table 1 refers to it as AMAP.

11) Fig. 3: Lower panel. Is MDP for TM or GEM?

12) Fig.7-9: Is Hg2+ different from RM?

13) Figs. 8-9: Units for $\mathrm{Hg} 2+$.

14) Fig. 8: Should the $x$-axis label be MDP instead of PMB?

Interactive comment on Atmos. Chem. Phys. Discuss., doi:10.5194/acp-2016-1074, 2016. 